\title{
Resistance oscillations in a Josephson-junction array in a magnetic field
}

\author{
J. M. Kosterlitz and E. Granato* \\ Department of Physics, Brown University, Providence, Rhode Island 02912
}

(Received 21 February 1986)

\begin{abstract}
An interpretation is proposed of the oscillations in the lower envelope of resistance as a function of magnetic field in the Josephson-junction array of Voss and Webb. We show that these variations can be explained by the presence of two incommensurate fundamental areas and of randomness in the array.
\end{abstract}

Ever since the original experiments ${ }^{1,2}$ were performed on the effects of a transverse magnetic field on the superconducting properties of a two-dimensional array of niobium tunnel junctions there have been two qualitative features of the data which have defied explanation. The first is the failure of the resistance minima at a finite number of flux quanta per elementary area to reach the zero-field value $^{3}$ and the long-period oscillations of the lower envelope of resistance. The second is that, beyond some critical field, these resistance minima start to rise slowly and linearly at higher fields. This latter phenomenon can be explained by a small amount of positional disorder in the array. ${ }^{4}$ In this Rapid Communication, we focus on the first point and propose an explanation of the variation of resistance minima without disorder by performing a careful analysis of the array used in the experiments.,

This array consists of niobium superconducting crosses and squares of $1 \mu \mathrm{m}^{2}$ cross section laid out in the lattice of Fig. 1. The intersection of a cross with a square forms a Josephson junction of $1 \mu \mathrm{m}^{2}$ area which we will assume to be simultaneously large enough so that charging effects 5 can be ignored but sufficiently small so that the field dependence of the effective coupling across the junction is

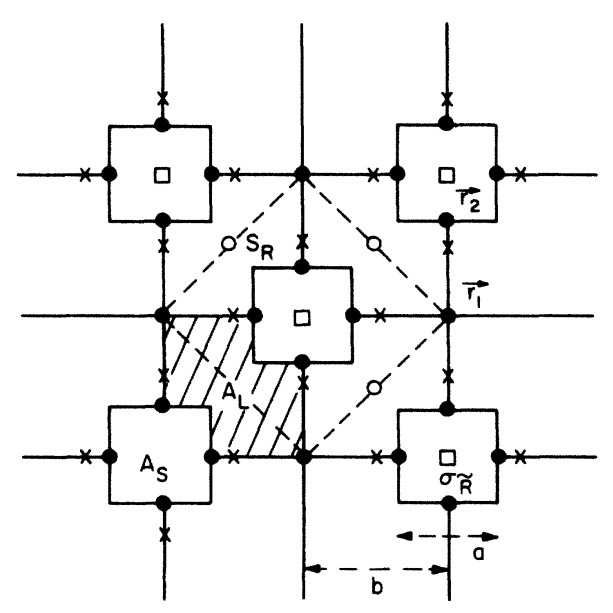

FIG. 1. The array analyzed in this work. Solid dots at $\mathbf{r}_{1}, \mathbf{r}_{2}$ represent the lattice of nodes of the superconducting network, crosses the weak links, and solid lines the superconducting wires. The dual lattice sites are denoted by open dots $(R)$ and squares $(\tilde{R})$. The two fundamental areas are the large squares $\left(A_{S}\right)$ and hatched area $\left(A_{L}\right)$. A unit cell is marked with a dashed line. negligible. The finite size of the junction is ignored in what follows. We make the further assumption that the superconducting elements are sufficiently thin so that flux can leak through them. Then the field is essentially uniform in the plane of the array and the system is always in thermal equilibrium. ${ }^{6}$ Under these physically reasonable assumptions, we can see immediately that there is another area in the problem besides the fundamental area $A_{L}$ formed by a loop containing four junctions. ${ }^{2}$ This latter area is responsible for the main oscillations of resistance since any defects in the flux lattice will be relatively mobile. The only energy barrier such defects have to overcome is the coupling across a junction. However, if this is the only area determining the period of oscillation, there can be no further variation of the resistance ${ }^{7}$ contrary to observation. We must therefore assume that the flux leaks in and out of the squares and this observation is the key to the problem.

In the original analysis ${ }^{2}$ of the array of Fig. 1, only the flux penetrating the area $A_{L}$ was taken into account which is responsible for the main oscillations with period $\Delta B$ $=\phi_{0} / A_{L}=0.17$ Oe. ${ }^{1}$ However, with the assumption that flux can leak across the superconducting wires, there is another fundamental area $A_{S}$, the area of a square, incommensurate with $A_{L}$. In order to obtain an absolute minimum in the resistance, the number of flux quanta $f_{L, S}=B A_{L, S} / \phi_{0}$ threading both $A_{L}$ and $A_{S}$ must be an integer; otherwise there will be mobile defects in the flux lattice dissipating the current. One can explain the observed variations in the resistance minima by these observations combined with the measured ratio of areas $A_{L} / A_{S}$ $=1.235$. ${ }^{1}$ Since the resistance is determined mainly by $f_{L}$ we concentrate on the minima at integer values of $f_{L}$. The deviation of the resistance from its absolute minimum at $B=0$ at higher integer values of $f_{L}$ will be determined by the deviation of $f_{S}$ from the closest integer and will be roughly proportional to this deviation. ${ }^{7}$ Now $f_{S}=f_{L} A_{S} / A_{L}=0.8097 f_{L}$ so that the deviations $\delta f_{S}$ of $f_{S}$ from an integer will be minima at certain values of $f_{L}$, where $f_{L}$ is restricted to integer values. For this ratio of areas, these occur at $f_{L}=0 \quad\left(\delta f_{S}=0\right), f_{L}=5,16$ $\left(\delta f_{S}=0.05\right), \quad f_{L}=10,11 \quad\left(\delta f_{S}=0.09\right), \quad$ and $\quad f_{L}=21$ $\left(\delta f_{S}=0.004\right)$, etc. Thus, one expects subsidiary minima in the lower envelope of resistance at these values of $f_{L}$ with the deepest one at $f_{L}=21$, the next deepest at $f_{L}=5,16$, and the highest at $f_{L}=10,11$. Of course, since the areas are probably incommensurate, the resistance 
variations are only quasiperiodic and are rather sensitive to the exact value of $A_{L} / A_{S}$ but this pattern fits the data very well.

To place these arguments on a firmer footing we now turn to a more careful analysis of the array of Fig. 1. We assume that we are at sufficiently low temperatures so that the magnitude of the superconducting order parameter is a position-independent constant and define the phase as $\phi\left(r_{1}\right)$ at the vertices of the crosses and $\theta\left(r_{2}\right)$ at the midpoints of the sides of the squares. The Hamiltonian of this system may be parametrized as

$$
\begin{aligned}
H / k T= & -\tilde{K} \sum_{\left\langle r_{1} r_{2}\right\rangle} \cos \left[\phi\left(r_{1}\right)-\theta\left(r_{2}\right)-A\left(r_{1} r_{2}\right)\right] \\
& -\tilde{L} \sum_{\left\langle r_{2} r_{2}^{\prime}\right\rangle} \cos \left[\theta\left(r_{2}\right)-\theta\left(r_{2}^{\prime}\right)-A\left(r_{2} r_{2}^{\prime}\right)\right],
\end{aligned}
$$

where

$$
A\left(r r^{\prime}\right)=\left(2 \pi / \phi_{0}\right) \int_{r}^{r^{\prime}} \mathbf{A} \cdot d \mathbf{l}
$$

and the integral is taken along the link joining the sites $r$ and $r^{\prime}$. In Eq. (1) we have introduced two parameters $\tilde{K}$ and $\tilde{L}$ to describe the coupling across a junction between nodes on a cross and a square $(\tilde{K})$ and between two nodes on a square $(\tilde{L})$ with $\tilde{K} \ll \tilde{L}$. Although the squares do not contain weak links, flux will leak in and out of the square so they are treated in the same way as the weak links but with a larger coupling constant. The choice of the nodes of the network is somewhat arbitrary but a different choice makes no difference to the final results. However, the recognition that the squares can be treated in the same way as the weak links is the key observation.

To obtain a gauge-invariant formulation, we follow the standard route by writing the partition function in the roughening representation ${ }^{8}$ on the dual lattice which makes clear the periodicities in this array and finally in the Coulomb-gas representation. As a first step, we approximate each term in the partition function by taking its Fourier representation ${ }^{9}$

$$
\begin{aligned}
& \exp \{-\tilde{K}[1-\cos (\phi-A)]\} \\
& \quad=\sum_{m} \exp \left[-m^{2} / 2 K-i m(\phi-A)\right],
\end{aligned}
$$

where, as usual, the Fourier coefficients are approximated by a Gaussian with $\tilde{K}=K$ for low temperatures. The roughening representation is straightforward to obtain with the result

$$
Z=\sum_{\{S, \sigma\}} \exp \left(-\frac{1}{2} K^{-1} \sum_{\left\langle R, R^{\prime}\right\rangle}\left(S_{R}-S_{R^{\prime}}\right)^{2}-\frac{1}{2} L^{-1} \sum_{\langle R \tilde{R}\rangle}\left(S_{R}-\sigma_{\tilde{R}}\right)^{2}-2 \pi i f_{L} \sum_{R} S_{R}-2 \pi i f_{S} \sum_{\tilde{R}} \sigma_{\tilde{R}}\right)
$$

where $f_{S}=B a^{2} / \phi_{0}$ and $f_{L}=B\left(b^{2}-a^{2} / 2\right) / \phi_{0}$ are the number of flux quanta through $A_{S}$ and $A_{L}$, respectively. When both $f_{L}$ and $f_{S}$ are integers this reduces to a complicated form of the standard roughening model. However, when either $f_{L}$ or $f_{S}$ is noninteger this becomes the discrete Gaussian model in an imaginary field whose properties are not well known. However, some information is available in the limit $L \rightarrow \infty$ and $f_{S}=0$ (Ref. 10) when the model reduces to a simpler but related model- the junction array on a square lattice with noninteger flux per plaquette. ${ }^{7}$ Numerical and analytic analysis showed that the critical temperature is a discontinuous function of $f_{L}$ and zero for $f_{L}$ irrational. More recent work has shown that in this case the system displays glassy behavior ${ }^{11}$ which will also lead presumably to metastability and hysteresis but such complications are not considered here. For this array, one expects that when either $f_{L}$ or $f_{S}$ is an integer, the other will be irrational or rational with a large denominator so that the system will be disordered at any finite temperature.

To make these arguments clearer and to see how the critical temperature depends on the two coupling constants $K$ and $L$, we map the system into the Coulomb-gas representation via the Poisson summation formula. To perform the resulting integrations it is convenient to divide the dual lattice into unit cells with three charges per cell as shown in Fig. 1. The resulting cells form a square lattice of spacing $b \sqrt{2}$ with charges at the vertices and centers of the bonds. The resulting Coulomb-gas Hamiltonian is

$$
\begin{aligned}
H_{c} / k T=2 \pi^{2} \sum_{i, j=1}^{3} \sum_{R, R^{\prime}}[ & \left.n_{i}(R)+f_{i}\right] \\
\times & {\left[n_{j}\left(R^{\prime}\right)+f_{j}\right] G_{i j}\left(R-R^{\prime}\right), }
\end{aligned}
$$

where $R$ denotes the cell sites (centers of squares), $i, j$ specify the intracell positions of the integer charges $n_{i}(R)$, and $f_{1}=f_{2}=f_{L}, f_{3}=f_{S} . G_{i j}(R)$ is a symmetric matrix, the Fourier transform of the inverse having elements

$$
\begin{aligned}
& G_{11}^{-1}(q)=G_{22}^{-1}(q)=\frac{4}{K}+\frac{2}{L}, G_{33}^{-1}(q)=\frac{4}{L}, \\
& G_{12}^{-1}(q)=-\frac{2}{K}\left[\cos \frac{1}{2}\left(q_{x}+q_{y}\right)+\cos \frac{1}{2}\left(q_{x}-q_{y}\right)\right], \\
& G_{13}^{-1}(q)=-\frac{2}{L} \cos \frac{1}{2} q_{x}, G_{23}^{-1}(q)=-\frac{2}{L} \cos \frac{1}{2} q_{y} .
\end{aligned}
$$

$G_{i j}(r)$ is logarithmically infinite with system size but

$$
\begin{array}{r}
G_{i j}(R)-G_{i j}(0) \simeq-[L K / \pi(2 L+K)] \ln \left|R / r_{0}\right|-E_{i j} \\
\text { for }\left|R / r_{0}\right| \gg 1 .
\end{array}
$$

The interaction between two widely separated charges is independent of the type of charges since this does not depend on their local environment. At first sight this leads to the wrong periodicity since this part of the interaction has a period $2 f_{L}+f_{S}=1$, where $2 f_{L}+f_{S}$ is the number of flux quanta in one cell.

To see where the separate periodicities resurface in this language, one must look at the short-ranged part of the interaction which feels the local environment of the charges. In this system, it is sufficient to calculate the fugacity matrix $y_{i j}=\exp \left(-E_{i j}\right)$ obtained by expanding $G_{i j}(q)$ to $O$ (1). In the physical limit $L / K \gg 1$, one obtains $E_{11}$ $=E_{22} \simeq E_{0}+3 K / 32, E_{33} \simeq E_{0}+L / 4$, and $E_{i j} \simeq E_{0}-K / 32$ for $i \neq j$, where $E_{0}$ is the usual core energy of the simple Coulomb gas, ${ }^{12}$ so that 


$$
H_{c} / k T=-[2 \pi L K /(2 L+K)] \sum_{i, j} \sum_{\substack{R, R^{\prime} \\\left(R \neq R^{\prime}\right)}}\left[n_{i}(R)+f_{i}\right]\left[n_{j}\left(R^{\prime}\right)+f_{j}\right] \ln \left|\left(R-R^{\prime}\right) / r_{0}\right|+\sum_{R} \sum_{i, j}\left[n_{i}(R)+f_{i}\right] E_{i j}\left[n_{j}(R)+f_{j}\right]
$$

We have ignored other short-range interactions coming from $O\left(q^{2}\right)$ terms in the expansion of $G_{i j}(q)$. These are important only if one wishes to study transitions at $f_{i}=n+\frac{1}{2}$, for example. ${ }^{7,13}$ Since the eigenvalues of $E_{i j}$ are all positive and distinct, the energy minima occur when each $f_{i}$ is an integer so that expected periodicity is restored by the short-range part.

One can draw a few conclusions from the form of Eq. (5). If $f_{L}$ and $f_{S}$ are both integers, the system reduces to three coupled Coulomb gases with integer charges which can be studied by standard renormalization-group methods ${ }^{9,12,14}$ and a single $X Y$ transition results at a critical point given by $\pi L K /(2 L+K)=1$. This corresponds to a resistive transition identical to that at zero field which is the standard one modified by finite-size effects. The resistance at other values of $f_{L}$ and $f_{S}$ will be controlled mainly by $f_{L}$ and will be roughly proportional to $\left|f_{L}-n\right|$ and will otherwise behave as discussed earlier. The observed resistance minima can be qualitatively explained by a simple form $R\left(f_{L}, f_{S}\right)=A\left|f_{L}-n_{L}\right|+B\left|f_{S}-n_{S}\right|{ }^{7}$ where $A \gg B$ are proportional to the mobilities of the defects in the flux lattice. With the measured ratio of areas $A_{L} / A_{S}=1.235$ we obtain remarkable qualitative agreement with the observed resistance minima at integer $f_{L}{ }^{15}$

When the field is increased further, randomness in the positions of the nodes of the superconducting network measured by a parameter $\Delta$ leads to an equivalent model of charges in a background of random dipoles with a distribution of width $B \Delta^{4}$ This model leads to the result that a superconducting phase exists for sufficiently small fields. Putting these results together yields a lower envelope of resistance in good qualitative agreement with experiment but a detailed comparison requires a much more sophisticated theory. The experimental observations that the deep minimum in resistance at $f_{L} \simeq 21-25$ is correlated with the beginning of the monotonic rise of the lower envelope must be attributed to coincidence since the critical field and the exact value of $f_{L}$ at the minimum are sample dependent and independent of each other. However, the main features of quasiperiodicity and an overall increase are explained by these arguments. One negative conclusion is that rather little about this system is universal and the details are very sample dependent.

The authors would like to thank R. A. Pelcovits for several useful suggestions and discussions and N. Read for discussions. J.M.K. acknowledges support from the $\mathrm{Na}$ tional Science Foundation under Grant No. DMR-8305022 and E.G. from the Conselho Nacional de Desenvolvimento Científico e Tecnológico (CNPq, Brazil).
"Permanent address: Instituto de Pesquisas Espaciais, São José dos Campos, S.P. 12200, Brazil.

${ }^{1}$ R. F. Voss and R. A. Webb, Phys. Rev. B 25, 3446 (1982).

${ }^{2}$ R. A. Webb, R. F. Voss, G. Grinstein, and P. M. Horn, Phys. Rev. Lett. 51, 690 (1983).

${ }^{3}$ In the array of Refs. 1 and 2 there are only about 20000 junctions and finite-size effects will dominate the resistance at zero field since at low $T$ the correlation length is larger than the system size.

${ }^{4}$ E. Granato and J. M. Kosterlitz, Phys. Rev. B 33, 6533 (1986); M. Rubinstein, B. Shraiman, and D. R. Nelson, ibid. 27; 1800 (1983).

${ }^{5}$ E. Simanek, Solid State Commun. 31, 419 (1979); Phys. Rev. B 23, 5762 (1981); K. B. Efetov, Zh. Eksp. Teor. Fiz. 78, 2017 (1980) [Sov. Phys. JETP 51, 1015 (1980)]; P. Fazekas, Z. Phys. B 45, 215 (1982); S. Doniach, Phys. Rev. B 24, 5063 (1981); J. V. José, ibid. 29, 2836 (1984).

${ }^{6} \mathrm{~A}$ partial Meissner effect for the squares is probable which would lead to hysteresis and metastability but a total Meissner effect would exclude the effect we are trying to explain.

${ }^{7}$ S. Teitel and C. Jayaprakash, Phys. Rev. Lett. 51, 1999 (1983); Phys. Rev. B 27, 598 (1983).

${ }^{8}$ S. T. Chui and J. D. Weeks, Phys. Rev. B 14, 4978 (1976).
${ }^{9}$ J. V. José, L. P. Kadanoff, S. Kirkpatrick, and D. R. Nelson, Phys. Rev. B 16, 1217 (1977); H. J. F. Knops, Phys. Rev. Lett. 39, 766 (1977).

${ }^{10} \mathrm{This}$ limit is also equivalent to assuming a complete Meissner effect for the squares when $f_{L}=B b^{2} / \phi_{0}$

${ }^{11}$ T. C. Halsey, Phys. Rev. Lett. 55, 1018 (1985).

12J. M. Kosterlitz and D. J. Thouless, J. Phys. C 6, 1181 (1973); J. M. Kosterlitz, ibid. 7, 1046 (1974).

${ }^{13}$ This problem has been studied by many authors with mixed success: W. Y. Shih and D. Stroud, Phys. Rev. B 32, 158 (1985); B. Berge, H. T. Diep, A. Ghazali, and P. Lallemand (unpublished); T. C. Halsey, Phys. Rev. B 31, 5728 (1985); J. Phys. C 18, 2437 (1985); M. Y. Choi and S. Doniach, Phys. Rev. B 31, 4516 (1985); M. Y. Choi and D. Stroud, ibid. 32, 5773 (1985); E. Granato and J. M. Kosterlitz, ibid. 33, 4767 (1986); J. Phys. C 19, L59 (1986); M. Yosefin and E. Domany, Phys. Rev. B 32, 1778 (1985).

${ }^{14}$ D. R. Nelson and B. I. Halperin, Phys. Rev. B 21, 5312 (1980).

${ }^{15}$ Experiment also shows minima at other rational values of $f_{L}$ but this is still not well understood, especially in the effects of competing periodicities and randomness. In this paper, these subsidiary minima are not considered. 